\title{
25 Research Soure \\ Heritability of familial pancreatic cancer in a Danish national family cohort
}

\section{Ming Tan ( $\nabla$ ming.tan@rsyd.dk)}

University of Southern Denmark: Syddansk Universitet

Klaus Brusgaard

University of Southern Denmark: Syddansk Universitet

Anne-Marie Gerdes

Rigshospitalet

Michael Bau Mortensen

University of Southern Denmark: Syddansk Universitet

Sönke Detlefsen

University of Southern Denmark: Syddansk Universitet

Ove B Schaffalitzky de Muckadell

University of Southern Denmark: Syddansk Universitet

Maiken Thyregod Joergensen

University of Southern Denmark: Syddansk Universitet

\section{Research}

Keywords: familial pancreatic cancer, heritability, family design, intraclass correlation

Posted Date: January 12th, 2021

DOI: https://doi.org/10.21203/rs.3.rs-141845/v1

License: (1) (1) This work is licensed under a Creative Commons Attribution 4.0 International License.

Read Full License 


\section{Abstract}

Background: Familial Pancreatic Cancer (FPC) is responsible for up to $10 \%$ of all cases of pancreatic ductal adenocarcinoma (PDAC). Individuals predisposed for FPC have an estimated lifetime risk of 18$38 \%$ of developing PDAC. While heritability of PDAC has been estimated to be $36 \%$ in a Nordic twin study, no estimate on FPC has been reported.

Methods: A national cohort of 27 Danish families with disposition for FPC is currently included in a screening program for PDAC at the Department of Medical Gastroenterology, Odense University Hospital. Familial disposition for FPC is defined by presence of either: A) at least 2 PDAC cases among first degree relatives (FDR) with at least one of the cases debuting $<50$ years of age, or $B$ ) at least 3 PDAC cases among FDRs. Family members included in the screening program were interviewed and the following data were obtained: cases of PDAC among FDRs ( e.g. , familial relation, age at diagnosis of PDAC), number of affected/unaffected siblings. Data were validated and supplemented with pedigrees for each FPC family obtained from the relevant departments of clinical genetics in Denmark. Heritability for FPC in FDRs of the predisposed families was estimated using a generalized mixed effect model, with calculation of an intra class correlation coefficient (ICC).

Results: Among 27 Danish families with predisposition for FPC, 83 cases of PDAC were identified. Eightyone of the PDAC cases were diagnosed as index cases in the respective families. The median age at diagnosis of PDAC was 66 years, and median time from diagnosis to death was 7.6 months. A total of 344 individuals were found as healthy FDRs of the 81 PDAC cases. The retrieved FDRs included a total of 230 individuals in sibship and 315 individuals in parent-offspring relatedness. We estimated an ICC of 0.25 (95\% Cl: $0.17-0.33)$, corresponding to a narrow sense heritability estimate of 0.51 (95\% Cl: 0.35-0.66) in the FPC family cohort.

Conclusion: The estimated heritability of $51 \%$ prominently underlines the genetic component of FPC. Our findings serve as a solid basis for conducting molecular genetic studies to look for genetic variations contributing to the development of FPC.

\section{Full Text}

This preprint is available for download as a PDF.

\section{Figures}


(a)

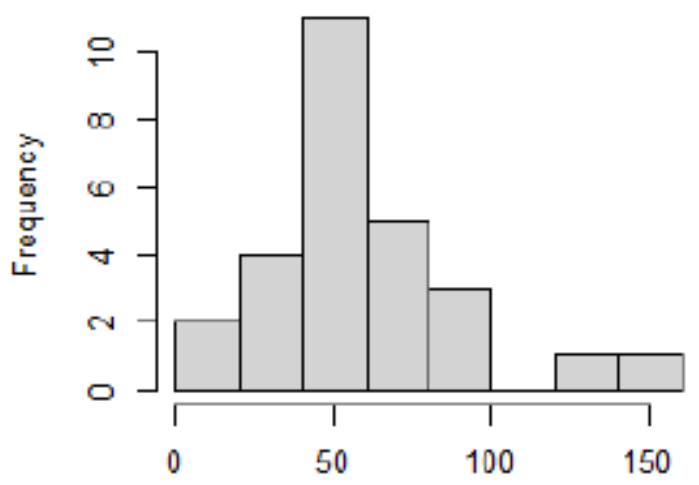

Pedigree size

(c)

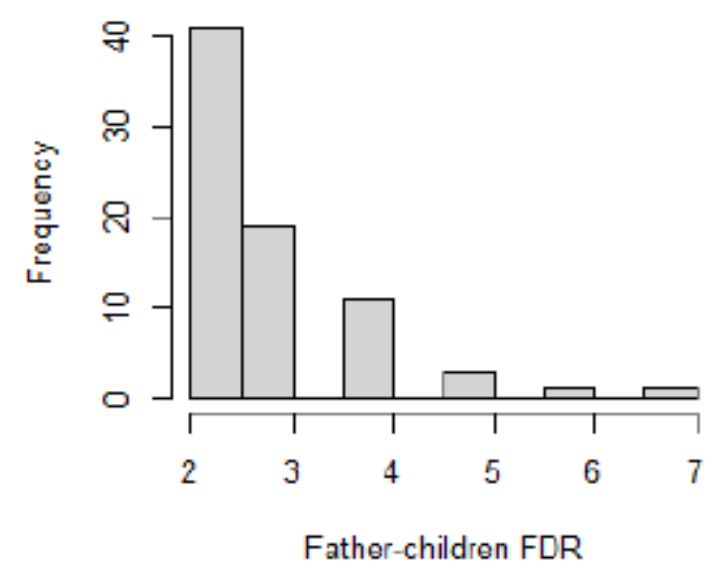

(b)

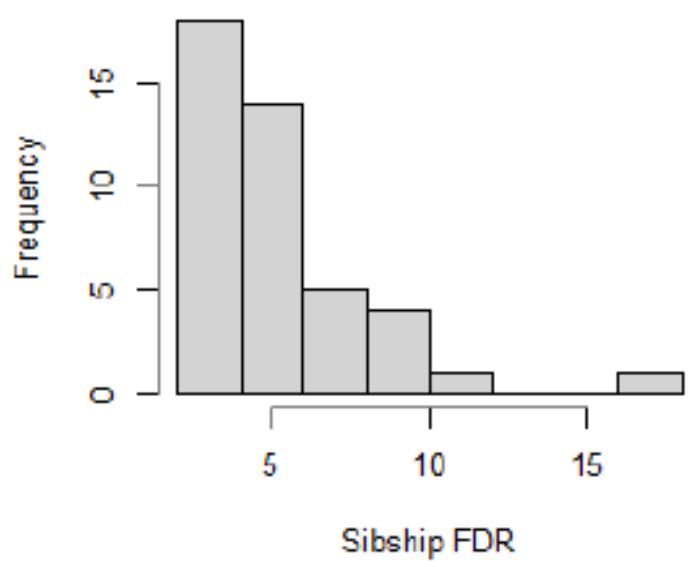

(d)

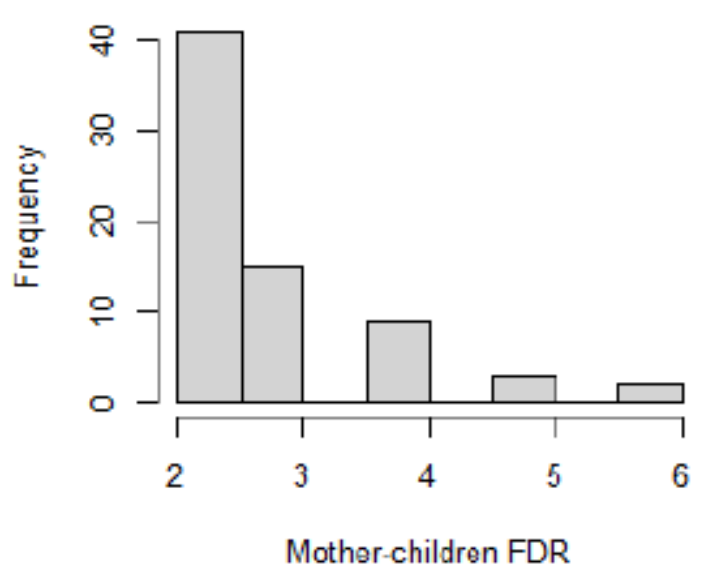

\section{Figure 1}

Histogram for the distribution of pedigree size (1a), sibship size (1b) and parent-offspring relatedness (1c-1d). 
(a)

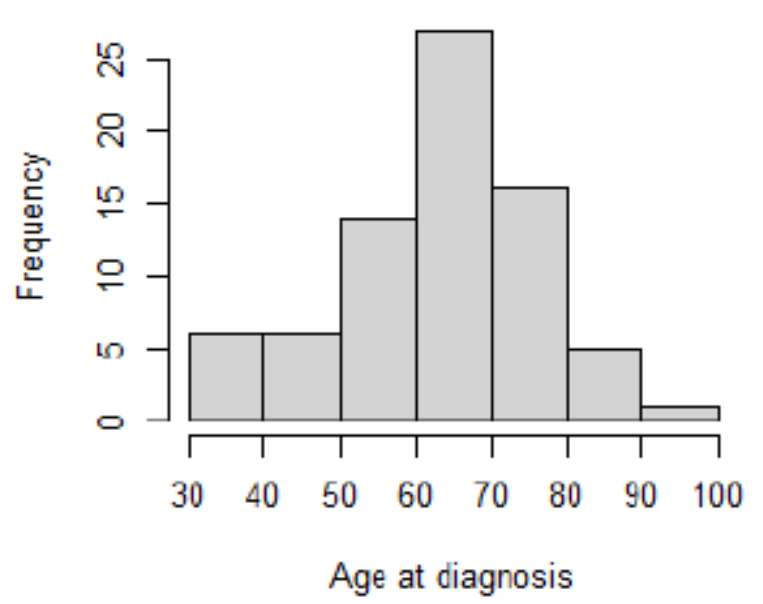

(c)

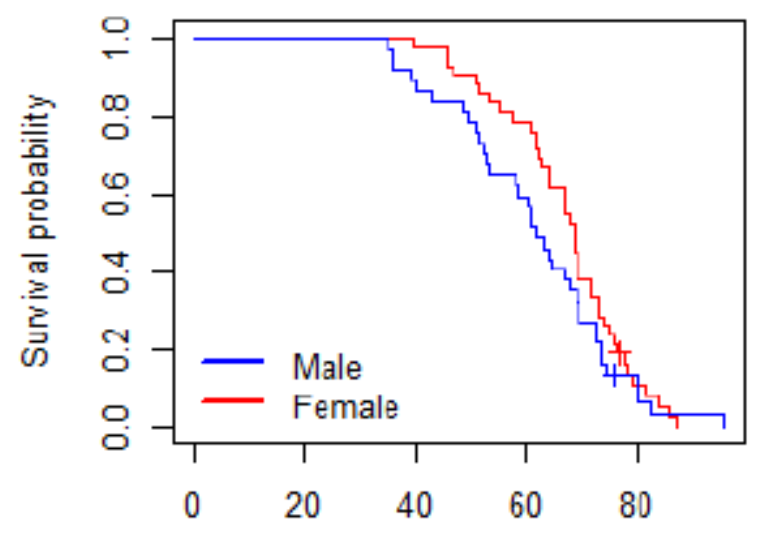

Age at death (b)

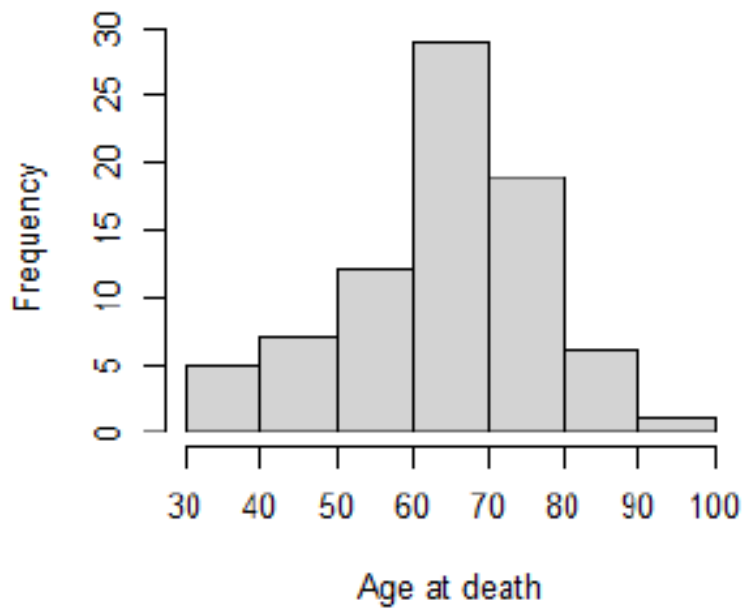

(d)

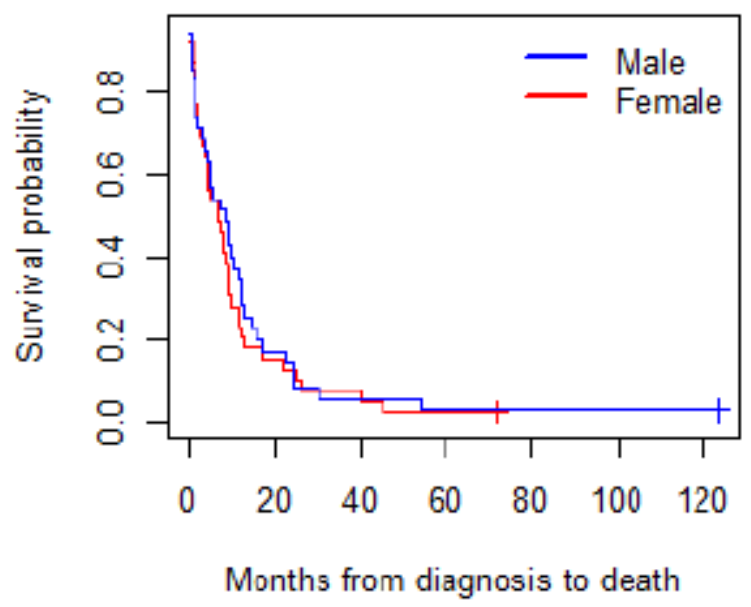

\section{Figure 2}

Histogram for median age at PDAC diagnosis (2a) and median age at death (2b). Kaplan-Meier plot for age at death (2c) and survival time from diagnosis to death (2d), for male (blue) and female (red) PDAC patients. 


\section{Median: $0.25,95 \% \mathrm{Cl}: 0.17-0.33$}

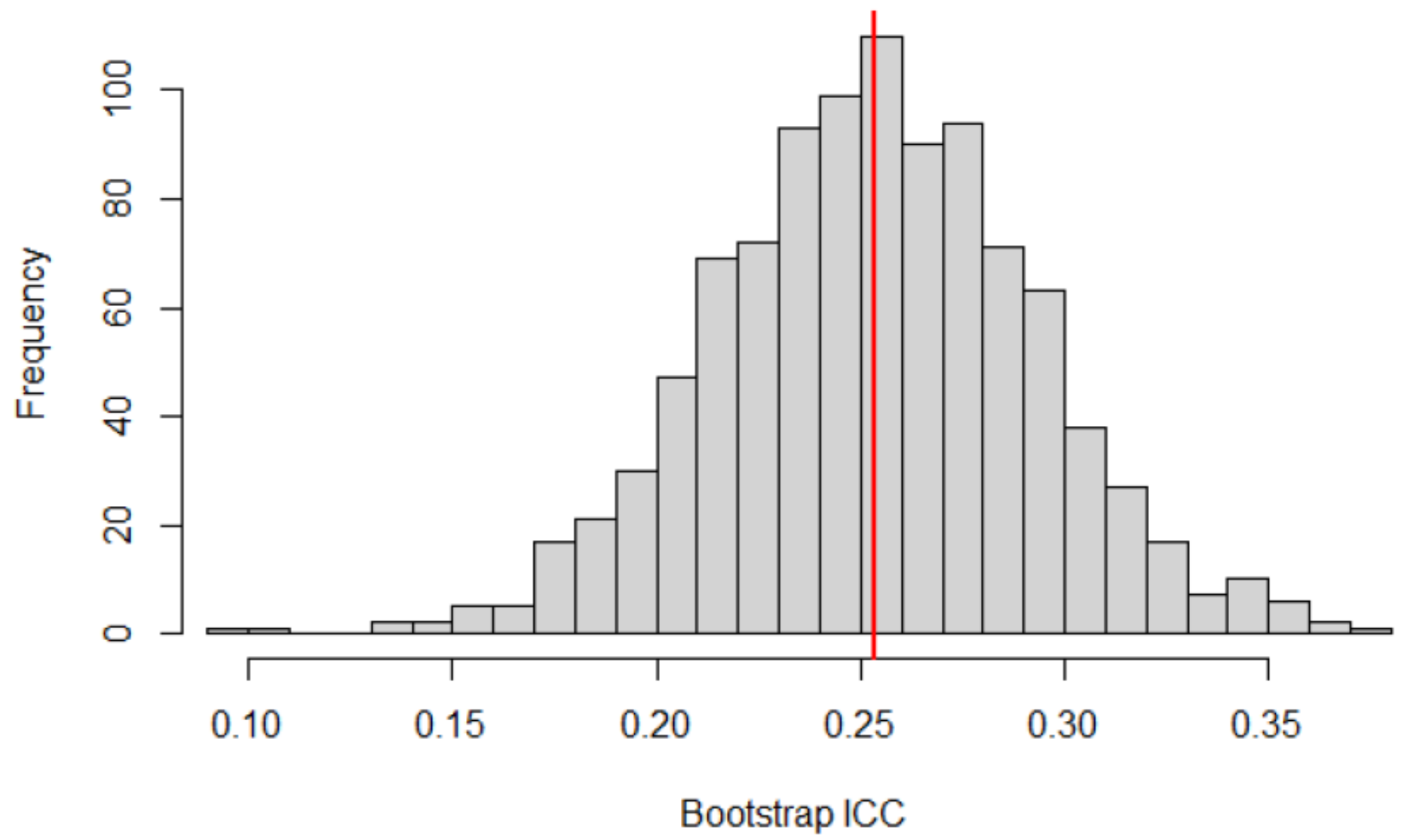

Figure 3

Histogram for the distribution of bootstrap ICCs. 